\title{
Cien años de autonomía universitaria latinoamericana
}

Recibido: 1 de septiembre 2019

Revisado: 3 de octubre 2019

Aprobado: 15 de noviembre 2019

\section{Juan Antonio Gutiérrez}

Slon

Costarricense. Máster en Historia en la Universidad de

Costa Rica. Bachiller en

Sociología en la Universidad

Nacional de Costa Rica. Investigador del Centro en Investigación en Cultura y

Desarrollo y del Centro

Agenda Joven de la

Universidad Estatal a

Distancia.

Correo electrónico: juanguts@gmail.com

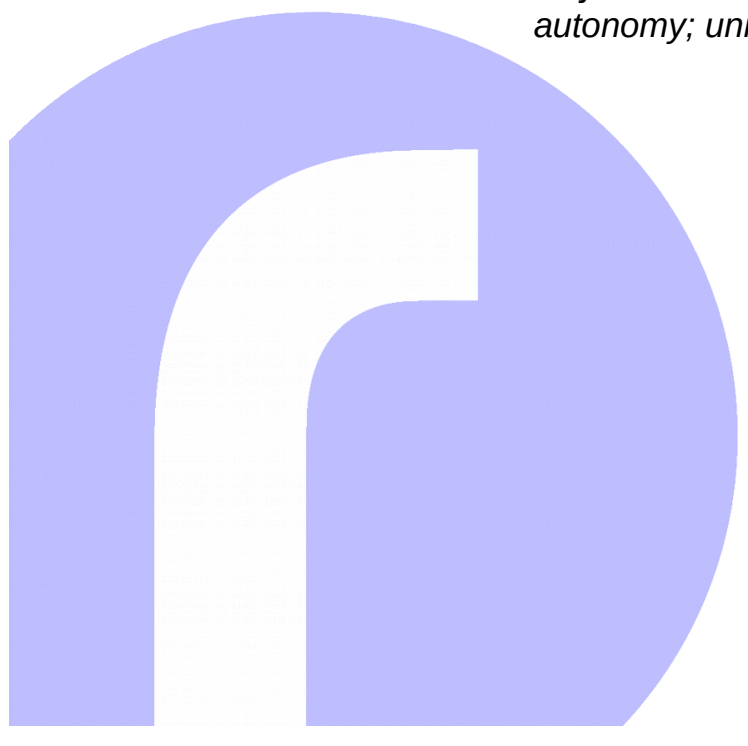

https://revistas.uned.ac.cr/index.php/rupturas/ (c) (1) (2)
Resumen: En el contexto del centenario de la Reforma Universitaria de Córdoba 1918-2018, se lleva a cabo un estudio basado en los principales planteamientos que sobre la autonomía universitaria se discuten en el campo académico latinoamericano. Desde un análisis sumario de este campo se caracteriza, interpreta y explica sobre los ámbitos de la autonomía universitaria como pilar democrático, espacio jurídico de poder diferenciado y la manifestación de las universidades públicas latinoamericanas como actores sociales presentes en un siglo lleno de experiencias políticas en torno a la constitución, la continuidad, los retos y la defensa de la autonomía universitaria.

Palabras clave: movimiento estudiantil; participación estudiantil; autonomía educativa; estudiante universitario; América Latina

\section{One Hundred Years of Latin American University Autonomy}

Abstract: In the context of the centenary of the University Reform of Córdoba 1918-2018, this study is based on the main approaches to university autonomy discussed in the Latin American academic field. From a summary analysis of this subject, the fields of university autonomy are characterized, interpreted and explained as a democratic pillar, legal space of differentiated power and the manifestation of Latin American public universities as social actors present in a century full of political experiences around the constitution, continuity, challenges and defense of university autonomy.

Key words: Student movements; student participation; educational autonomy; university students; Latin America 


\section{Introducción}

La autonomía universitaria moderna es un movimiento latinoamericano iniciado en 1918 y que fue adquiriendo un importante protagonismo en la realidad social y política del continente. Surgió como protesta contra la conducción conservadora, eclesiástica, militar y autoritaria de la universidad heredada desde la Colonia. La reforma de la autonomía universitaria es un movimiento político que se fue propagando en América Latina según el posicionamiento que sobre esta iban realizando estudiantes, docentes y personal administrativo en cada universidad.

Su principal aspiración es la conquista del gobierno interno de la universidad desde una lógica de organización que se geste según las necesidades, los contextos y las dinámicas propias de cada centro de educación superior.

De esta manera, el artículo se divide en cuatro apartados que explican los alcances de esta expresión política, social y jurídica desde la cual las universidades latinoamericanas se han posicionado frente los diferentes Gobiernos y Estados, argumentando una separación con estos en función de mantenerse rectoras de sus políticas internas, contenidos curriculares, matrícula, investigación, gestión, acción social, administración financiera y alternabilidad representativa interna.

El primer apartado está compuesto por tres subapartados y es dedicada al acontecer histórico sobre la conquista de la autonomía universitaria entendida como la capacidad de los estudiantes universitarios, docentes y administrativos para constituirse como cuerpos políticos que los representa dentro de la universidad y les permita desplegarse hacia el entorno político nacional e internacional, con énfasis en un acercamiento al contexto de América Central. En el segundo apartado, se reflexiona sobre la figura del cogobierno universitario en su relación autónoma con los Estados y los poderes de las Repúblicas, asumiendo los derechos políticos constitutivos de la reforma universitaria.

Seguidamente, en el tercer apartado se reflexiona sobre los retos actuales de la autonomía universitaria en el contexto de privatización de la educación superior, el énfasis gerencial que el neoliberalismo promueve y la necesidad de reforzar las consignas de la reforma, en un ámbito latinoamericano de amplias desigualdades sociales. Por último, se concluye con reflexiones sobre el centenario de la Reforma Universitaria de Córdoba 1918-2018 y su continuidad desde una postura académica en defensa del legado de Córdoba. 


\section{El sentido nosotros-universitario y la construcción política de la autonomía}

La definición de la autonomía como baluarte de la universidad, estipulada desde lo jurídico y la relación institucional con el Estado, se refuerza desde la constitución de una colectividad universitaria como poder político. Esta es la expresión misma de la autonomía universitaria que se ha venido construyendo en varios momentos desde las expresiones académicas, económicas, sociales y políticas inscritas en la relación Estado-Sociedad-Universidad.

Esta cuestión es central para Naishtat, Raggio, Schuster y Villavicencio (1996), quienes en su artículo Filosofía política de la autonomía universitaria argumentan que "sin un claro significado de la autonomía, la universidad pública no podría ser ella misma la protagonista y el sujeto de su transformación" $(1996,2)$.

Tal definición, desde la propia universidad, lleva implícita la discusión sobre las definiciones mismas de la autonomía como elemento constitutivo en la creación de las universidades públicas latinoamericanas; se trata de modelos que según estos autores serían (Naishtat et al. 1996, 2):

$\checkmark$ El modelo profesionalista, que responde a la competencia profesional.

$\checkmark$ El modelo científico, que promueve el ideal de ciencia y saber teórico.

$\checkmark$ El modelo humanista, basado en la noción de universidad como centro cultural.

$\checkmark$ El modelo ético democrático, cuya finalidad es la educación de la ciudadanía.

Estos modelos estarían desde la propia instauración de la autonomía como una respuesta a la imposición de criterio de formación universitaria y el ascenso, en 1918, de una modelo de universidad como un "sujeto político ilustrado, como intelectual colectivo y crítico (Naishtat et al. 1996, 2).

Este sujeto político llamado "autonomía universitaria" ha construido una noción de identidad universitaria posicionándola como colectivo histórico frente a los poderes del Estado. Esa relación ha sido bien expuesta por la autora mexicana María Herlinda Suárez Zozaya en su artículo Consideraciones políticas sobre la autonomía universitaria (2010), en el cual expone que hay una construcción de un "Nosotros-Universidad" que se opone a un "Otro-Estado".

La autora plantea una significación en la relación histórica y política que surge con la reforma de la Universidad Mayor de San Carlos y Monserrat de Córdoba en 1918, lo que constituyó un reclamo de pluralismo social y una acotación del poder del Estado eclesiástico y militar (Suárez 2010, 28).

En esta concepción del "Nosotros-Universitario" frente al "Otro-Estado", los movimientos estudiantiles estarían agenciados como parte del conjunto universitario pero con expresiones y acciones propias que plantean otro nivel de autonomía desligado de la capacidad de acción que emerge desde el Cogo- 
1. La Autonomía Universitaria y el Cogobierno Estudiantil son elementos indisolubles pues la primera surge como consecuencia de las protestas de jóvenes universitarios desde la creación de la Federación Universitaria Argentina el 11 de abril de 1918, que se posicionó como modelo ejemplar a partir de junio de 1918, cuando se establecieron las condiciones de la autonomía universitaria y la correlación de fuerzas políticas tanto al interno como externo de la Universidad. bierno Universitario; el cual desde sus inicios postuló una praxis de democratización del espacio político universitario bajo el emblema de que "el demo universitario, la soberanía, el derecho a darse el gobierno propio radicaba principalmente en los estudiantes", hecho que evidenciaba "las demandas de la nueva generación" (Suárez 2010, 35).

Aquel preciso momento fue el auge de un sentido propio de autonomía universitaria que implicó haber "ganado derechos y también adquirido responsabilidades; y comprendió que más allá del otorgamiento legal, la autonomía universitaria tenía un carácter legítimo fincado en el ejercicio de un poder moral" (Suárez 2010, 35).

Dicha disputa es parte perenne de la ratificación del "Nosotros-Universitario", visto como la facultad de las universidades autónomas de mediar la diversidad ideológica y política que confluyen en el marco de los diferentes manifestaciones de poder universitario consignado en las instancias académicas, estudiantiles, administrativas y sindicales, que pueden actuar como uno o varios bloques políticos, frente la construcción de otredades políticas que se encuentran en el Estado, el mercado y la institucionalidad internacional (Suárez 2010, 40).

El esquema en el cual la autonomía universitaria se presenta como instancia unitaria para la construcción de identidades políticas universitarias frente a otras instancias nacionales o internacionales, es que se entiende el alcance amplio y diverso de la autonomía universitaria en razón de su dimensionalidad jurídica, económica, política, social y filosófica que la constituyen como un espacio de poder diferenciado.

Desde esta la reflexión sobre autonomía universitaria como espacio de poder, se puede comprender los alcances y las condiciones inherentes de la educación superior, aspecto que es central en el artículo El principio de autonomía universitaria entre Escila y Caribdis (2011), de Luis Muñoz Varela y Juan Félix Castro Soto.

Ambos autores vinculan la autonomía como cualidad política de las universidades públicas en su relación con el Estado y demás actores sociales, en el momento que construyen relaciones entre lo que la autonomía posibilita en su relación con la esfera de la educación superior como tal y diferenciada de otras plataformas educativas.

Muñoz y Castro $(2011,10)$ diferencian cuatro aristas que integran esta relación entre autonomía y educación superior: la función en cuanto al trabajo profesional; la diferenciación académica universitaria de otras docencias; la demanda que sobre la educación superior existe; y la relación de autonomía como parte de las políticas públicas referentes a la educación.

De modo que la sola presencia del principio de autonomía como principio de institucionalidad no debe entenderse, afirman Muñoz y Castro $(2011,28)$, únicamente desde el marco constitucional y normativo, sino como "la puesta en práctica y validación de este insoslayable principio". 
La reflexión dada por estos autores invita a un análisis situado de la autonomía

universitaria tomando en cuenta su carácter de historicidad y las relaciones

interinstitucionales en el marco general de las tendencias de los Estados en

Latinoamérica, situación que se discute en el siguiente subapartado.

\section{La latinoamericanidad de la autonomía universitaria}

El debate sobre los alcances y elementos constitutivos de la autonomía universitaria presenta un importante registro histórico en el contexto de Latinoamérica, escenario en el que surgió y se ha tenido que defender para mantenerse vigente. En este aspecto, la lucha por impulsar la autonomía universitaria frente a los diferentes Estados del primer cuarto del siglo XX dado por el movimiento reformista, adquirió niveles de movimiento social al sobrepasar los alcances de la universidad misma. Así se expone en el artículo Historia de la autonomía universitaria en América Latina, de la especialista en el tema, la mexicana Renate Marsiske Schulte $(2004,165)$, que explica que en Perú, durante las décadas de 1920 y 1930, el movimiento reformista propició el aumento de la organización sindical más allá de la universidad; y paralelo a las luchas de índole estudiantil y universitario, se aliaron a luchas por derechos laborales.

Marsiske (2004) argumenta que para el caso del movimiento estudiantil en Cuba -en estas mismas décadas- se emprendieron acciones de formación entre estudiantes y obreros, lo que fungió como "universidades populares" con un alto contenido político relacionado con el saber científico, tecnológico, cultural y letrado que se estudiaba como parte de una agenda de transformación social de mayor envergadura, que en el caso cubano implicaba luchar por una independencia plena ${ }^{2}$.

2. Tanto para el caso de Perú como el de Cuba, la dirigencia

En aquel escenario de organización alrededor de la autonomía universitaria las décadas de 1920, 1930 y 1940 fueron teniendo matices autónomos de acuerdo con las condiciones políticas de cada país latinoamericano. Hubo eventos propios de la acción estudiantil universitaria que fueron profundizando el movimiento reformista mediante encuentros nacionales e internacionales de estudiantes universitarios; hechos que explica el autor Augusto Cazali Ávila en su artículo La autonomía universitaria en Centroamérica $(1977,14)$ y en el que evidencia la creciente organización universitaria mediante recurrenliderazgo social donde surgieron dirigentes estudiantiles que marcarían la historia de sus naciones y del continente, tales como Raúl

Haya de la Torre y José Carlos Mariátegui -en el caso peruano-y Julio Antonio Mella -en el caso cubano. tes congresos estudiantiles de carácter internacional como:

el Congreso Iberoamericano de Estudiantes (México, 1931); el Congreso de la Universidad Boliviana (Sucre, 1941); y el II Congreso Iberoamericano de Estudiantes (Costa Rica, 1933) fijaron criterios más decididos sobre la autonomía universitaria, definiéndola como: "derecho a 
elegir sus autoridades, darse sus reglamentos, dictar sus

planes de estudios, preparar su presupuesto y orientar la enseñanza con independencia del Estado.

Tales congresos estudiantiles implicaron la comunicación articulada de organizaciones estudiantiles que venían abriendo espacios de representación en diferentes universidades de América Latina, siguiendo un movimiento de expansión al propiciar espacios unitarios desde diferentes asociaciones y federaciones estudiantiles. En ese sentido, Cazali $(1977,14)$ menciona que:

La afirmación más amplia y definitiva sobre la autonomía universitaria en Latinoamérica se dio en el Primer Congreso de Universidades Latinoamericanas, reunido en Guatemala en 1949, durante el cual se aprobó la Carta de dichas Universidades, ratificada con algunas reformas por el Segundo Congreso, realizado en Chile en 1953.

Con este tipo de encuentros, las juventudes universitarias latinoamericanas fueron impulsando la autonomía como consigna para el cambio social. Entonces, se optaba por espacios gremialistas en función de procurar un movimiento educativo autónomo en todo el continente. Intención y contexto en los cuales los estudiantes universitarios de América Central cumplieron un activo protagonismo durante las décadas de 1930 y 1940, situación que repasaremos en el siguiente subapartado.

\section{La autonomía universitaria en América Central}

La participación de organizaciones universitarias que impulsaron el proceso de autonomía desde América Central tuvo su momento de expansión en la década de 1940, lo que se consideró como un proceso tardío en relación con otros países de América del Sur que tuvieron su apogeo en la década de 1920.

Según Cazali $(1977,11)$, el proceso tardío en la subregión estuvo relacionado con el entrabamiento que Gobiernos autoritarios dieron al desarrollo educativo, con la inestabilidad política y con conflictos entre las naciones en las primeras tres décadas del siglo XX, pese a ello, entre las décadas de 1920 y 1930 también existieron asociatividades de jóvenes universitarios dirigidos bajo agendas gremiales muy puntuales que no lograron un proceso reformista universitario en profundidad ${ }^{3}$.

Sin embargo, para América Central la década de 1940 representó un periodo de lucha, organización y demanda de autonomía universitaria como un proceso sociopolítico que estuvo cercano a otras acciones civiles, sociales y políticas. Sobre tal contexto Cazali $(1977,16)$ explica que en el caso de El Sal- 
vador y Guatemala los movimientos de lucha contra las dictaduras ocurrieron de la mano con los procesos de lucha por la autonomía universitaria.

Durante esa década de conflicto político y cambio social, se llevó a cabo el Primer Congreso Centroamericano de Universidades, reunido en El Salvador en setiembre de 1948, y en el que hubo participación de la Universidad de El Salvador (UES), la Universidad de Costa Rica (UCR), la Universidad de San Carlos de Guatemala (USAC) y la Universidad de Honduras (UH), además de una representación en calidad de observadores por parte de la Universidad Libre de Nicaragua, la Universidad de Panamá (UP) y la Universidad de Caracas, desde Venezuela (Cazali 1977, 16-17).

Como un importante resultado de este congreso se considera el intercambio que estudiantes, académicos y autoridades universitarias realizaron en 1948, situación que según Cazali $(1977,17)$ sirvió como "germen de posteriores transformaciones", no solo en lo concerniente al ámbito universitario, pero sí con cierta centralidad. A su vez, entre los principales acuerdos de este congreso estuvieron:

que cada Universidad del Istmo, adquiriera o reforzara su autonomía, por lo menos en los aspectos básicos siguientes: 1) la facultad de autonormarse; 2) la libertad de elegir a sus autoridades, sin interferencias externas; 3) la posibilidad de elaborar sus planes y programas, por decisión propia; y, 4) la obtención o establecimiento de un patrimonio propio, y su libre manejo, sujeto al mínimum de controles externos (Cazali 1977, 19).

Este Congreso Centroamericano -al igual que los ya citados realizados en el contexto de América Latina- fue parte de las expresiones colectivistas juveniles estudiantiles que fueron dando forma al proceso de reforma universitaria en la región. Sin embargo, no todos los procesos implicaron un trabajo de red internacional. Tal cual fue el caso panameño que se reseña en el artículo En defensa de la autonomía universitaria del exrector de la UP, Gustavo García de Paredes $(2008,48)$, referido a las acciones estuvieron acordes con el devenir histórico de este país:

La Universidad de Panamá, fundada en 1935, única de carácter estatal hasta 1981, no fue ajena al movimiento reformista. El concepto influyó en las huelgas de 1942 y 1943, al igual que en las legislaciones aprobadas a partir de 1946. En efecto, las reclamaciones de autonomía universitaria y estabilidad del profesorado, por parte de los 
estudiantes, a raíz del despido de dos profesores universitarios en los años 1942 y 1943, dieron lugar a la expedición del Decreto n. ${ }^{\circ} 720$ de 17 de noviembre de 1943, el cual otorgó un régimen de autonomía parcial a la Universidad de Panamá.

Este proceso de Panamá, ubicado en la década de 1940, lo hace coincidir con el resto de acciones reformistas de América Central, que protagonizaron en este decenio la etapa de formalización jurídica e institucional de la autonomía universitaria. De esta manera, los diferentes procesos para instauran la autonomía universitaria que sucedieron durante la primera mitad del siglo XX en América Central y América Latina fueron parte de los procesos sociales y políticos que ayudaron a acentuar los modelos de sociedad en el continente.

Todo lo anterior, como es de suponer, no estuvo ausente en el contexto de la historia de Costa Rica, ya que también vivió importantes procesos de cambios sociales y políticos durante la década de 1940, momento cuando se definieron las bases de la República que se instauraría en la segunda mitad del siglo $\mathrm{XX}$, y en el que las luchas estudiantiles y movimientos universitarios fueron parte fundamental para impulsar procesos de desarrollo social, político y democrático del país. En este contexto y sin generalizar, se pasará a ampliar sobre el caso costarricense, desde el cual se podrá comprender mejor lo que la autonomía universitaria latinoamericana conlleva.

\section{La autonomía universitaria en Costa Rica}

4. Una referencia en el que se pueden comprender las raíces de este proceso y en el que se reflexiona de forma pionera sobre estas temáticas es: Galdámez, Luis (1935). La universidad autónoma. San José, Costa Rica: Editorial Borrasé.

5. Así se explica en un documento que el Archivo de la UCR mantiene disponible en su sitio web:

http://archivo.ucr.ac.cr/ustot.ht $\mathrm{ml}$

y que se presenta al público mediante el título: "La Universidad de Santo Tomás (1843-1888)". Conversación con el Prof. Rafael Obregón Loría, Revista de Filosofía de la UCR 3 (9), 1961.
La reforma universitaria de Costa Rica que se profundizó en la década de 1940 y tuvo como eje articulador la creación ${ }^{4}$ de la Universidad de Costa Rica (UCR), hacia el inicio de esa década mediante la creación de la Ley Orgánica de la Universidad de Costa Rica en 1940, con la cual el país volvería a tener educación superior universitaria luego de varias décadas sin este tipo de enseñanza, que había entrado en descenso desde el cierre de la Universidad de Santo Tomás en $1888^{5}$.

Este vacío de universidades en Costa Rica cambió con el inicio del gobierno socialcristiano que en agosto de 1940 -mediante la Ley n. ${ }^{\circ}$ 362-impulsó la creación de esta institución; sin embargo, en sus primeros ocho años, la UCR mantenía una existencia "tradicional, por dos razones; en primer lugar, su principal objetivo es la formación de profesionales; y en segundo lugar, su ideología estaba fuertemente influenciada por las clases políticas imperantes", según explica el costarricense Vladimir Villalobos González $(2005,29)$ en su tesis de derecho Autonomía universitaria y aplicación del artículo 84 de la Constitución Política de Costa Rica, caso Universidad de Costa Rica, periodo 1990 a 2004.

Según este investigador, la UCR adquirió su grado de institución autónoma mediante su inclusión en la Carta Magna que surgió luego del conflicto políti- 
co armado de 1948 y el llamado a una Asamblea Constituyente en 1949, cuando se incluyó el artículo constitucional n. ${ }^{\circ} 84$ que da pie a la autonomía universitaria y que dicta lo siguiente:

ARTíCULO 84.- La Universidad de Costa Rica es una institución de cultura superior que goza de independencia para el desempeño de sus funciones y de plena capacidad jurídica para adquirir derechos y contraer obligaciones, así como para darse su organización y gobierno propios. Las demás instituciones de educación superior universitaria del Estado tendrán la misma independencia funcional e igual capacidad jurídica que la Universidad de Costa Rica. El Estado las dotará de patrimonio propio y colaborará en su financiación. ${ }^{6}$

6. Así reformado por Ley $n .^{\circ}$ 5697 del 9 de junio de 1975. Tomado de la web de la

Los alcances de la autonomía universitaria desde la Constitución Política incluyen las potestades que la universidad pública tiene respecto de otros poderes de la República como la Asamblea Legislativa, sobre lo que el investigador costarricense Hugo Alfonso Muñoz Varela $(1979,194)$ explica lo s guiente:
Universidad de Costa Rica en su sitio sobre documentos de importancia histórica, política, cultural o institucional: https://www.ucr.ac.cr/medios/d ocumentos/2015/constitucion politica.pdf

La Constitución permita, pues, que la Asamblea Legislati-

va dicte normas relativas a las materias "puestas bajo la competencia" de las Universidades (...) Es así como la Asamblea no puede modificar una norma universitaria en materias que son de la competencia (...) Al contrario, las universidades pueden modificar leyes en el campo académico cuando éstas hayan sido dictadas en ausencia de norma universitaria. El legislador puede dictar leyes en materia universitaria si no existe norma; si la hay, carece de potestad.

Con esta explicación de Muñoz, se torna evidente que la autonomía universitaria se ratifica frente a otras instancias de poder republicano, trazando una relación jurídico- institucional que sirve como regulador de la autonomía y emisor de esta. Así pues, en Costa Rica, la relación de poder entre la Asamblea Legislativa y la universidad pública del país se regula directamente desde la Carta Magna, en la cual, para los años de las décadas de 1950 y 1960, también estipulaba los grados de autonomía financiera. 
7. Desde del año 2010 se cambia su nombre a Tecnológico de Costa Rica (TEC).

8. La negociación del FEES cada año implicó que las agrupaciones estudiantiles y universitarias se movilizaran en varias ocasiones a finales de la década de 1970 e inicios de década de 1980. Fueron años de importantes movilizaciones de presión política por parte de la juventud universitaria costarricense.

9. Integrado inicialmente por la UCR, ITCR (actualmente TEC), UNA y UNED. Luego, con la creación de la Universidad Técnica Nacional (UTN) en el 2008, esta también se unió a CONARE.

10. En años recientes, 2010 , 2014, 2018 y 2019, varias asociaciones y federaciones estudiantiles emprendieron procesos de lucha en el marco de la negociación del FEES, incluyendo manifestaciones cierre de vías y tomas de edificios universitarios; en el 2010 y 2019 incluyó este tipo de acciones en UNA, UCR y TEC, tanto en sus sedes principales como en algunas de sus sedes.
Sobre este aspecto, Muñoz $(1979,203)$ explica que durante esos años se tenía tipificado el porcentaje de financiamiento al que la UCR tenía derecho: "[e]l constituyente se preocupó por la autonomía financiera y estableció en la propia Constitución, que el $10 \%$ del presupuesto general de la educación pública correspondía a la Universidad de Costa Rica".

Sin embargo, hacia la década de 1970 ese porcentaje resultó insuficiente debido a la creación de otras tres instituciones de educación superior universitaria estatales: el Instituto Tecnológico de Costa Rica ${ }^{7}$ (ITCR) 1971; la Universidad Nacional de Costa Rica (UNA), 1973; y la Universidad Estatal a Distancia (UNED), 1977; hechos que transformarían el modo de financiamiento e interinstitucionalidad entre los poderes de la República y las instancias de educación superior universitaria.

En ese nuevo escenario costarricense de la educación superior implicó que hacia 1976, y en buena parte debido a la presión por parte de estudiantes, docentes, administrativos y autoridades políticas de las universidades públicas, se implementaron reformas tributarias en cuanto al financiamiento de las universidades públicas del país. Se optó por un modelo en el cual -en un principio- anualmente se negociaría el presupuesto que cada universidad obtendría para la ejecución de sus funciones culturales, educativas y políticas (Muñoz 1979, 203).

En ese contexto, en 1976 se cambiaría de estipular 10\% del presupuesto nacional para la educación pública a constituir el Fondo Especial para el Financiamiento de la Educación Superior (FEES). Según explica Hugo Muñoz (1979, 203-204), el FEES inicialmente estuvo conformado por "el producto del Impuesto sobre el Traspaso de Bienes Inmuebles; y, además, por el 25\% hasta $30 \%$ de los ingresos del Impuesto sobre la Renta y por el producto del Impuesto sobre sociedades con acciones al portador".

Empero, la puntualización sobre presupuesto que anualmente recibirían las universidades dejó de ser fijo para pasar a un modelo de negociación anual ${ }^{8}$ en el que intervienen el Poder Ejecutivo y las universidades públicas del país mediante el espacio unitario del Consejo Nacional de Rectores (CONARE) ${ }^{9}$, gracias a la reforma del artículo 85 que se hizo a la Constitución Política en 1977 (Muñoz 1979, 204).

Este modelo de negociación del FEES pasó por otra modificación en 1982, cuando se comenzó a negociar de forma quinquenal. Así se mermaron las presiones estudiantiles para dicho fin y se resolvieron los problemas constantes con los estimados propuestos por el Gobierno y los negociados por el CONARE ${ }^{10}$.

Sobre este mismo contenido de vinculación entre autonomía y el presupuesto universitario, y siguiendo lo expuesto por la exrectora de la Universidad de Costa Rica, Yamileth González García, en su artículo El reto de la autonomía universitaria (2011), se explica que el "Convenio de Coordinación de la Educación Superior Universitaria Estatal en Costa Rica", en el que las rectorías de las cinco universidades públicas del país deben negociar su presupuesto universitario con la Presidencia de la República y los Ministerios de Educa- 
ción, Hacienda y Planificación es "una lucha desgarradora por mantener a las universidades costarricenses sirviendo al país"11 (González 2011, 35).

Si bien la autonomía es una facultad que en Costa Rica tienen las cinco universidades públicas (UCR, UNA, TEC, UNED y UTN), solo pocas ocasiones estas han emprendido ejercicios reflexivos y analíticos sobre esta. Lo anterior implica que la defensa de la autonomía se realiza más desde las acciones de hecho y de derecho, y menos desde los ejercicios académicos para su comprensión y protección.

En este aspecto, el artículo del sociólogo costarricense Daniel Camacho Monge, titulado La autonomía universitaria, la vigencia del III Congreso Universitario y una obligada referencia a Rodrigo Facio (2012), es una de las reflexiones primordiales sobre parte de la trayectoria histórica de la autonomía universitaria en la UCR.

El contenido que Camacho Monge expone se centra en los alcances que el III Congreso Universitario de la UCR obtuvo durante su realización entre 1973 y 1974; según el autor, fueron de avanzada y se mantienen con vigencia en la actualidad. Según Camacho $(2012,12)$, la raíz del congreso estuvo sustentado e inspirado por el estudiantado universitario en defensa de la autonomía:

La respuesta de la comunidad universitaria — digo bien 'la comunidad' porque surgió de las bases docentes y estudiantiles - fue la convocatoria del III Congreso, con el objetivo de realizar un auto examen institucional y renovar el rumbo de la Universidad, antes de permitir injerencias externas.

Esta resistencia frente la injerencia externa de la UCR -por entonces la única universidad del país- surgió como respuesta a la iniciativa del Gobierno que "insinuó la intención de revisar si la Institución ejecutaba políticas adecuadas al desarrollo del país y si invertía bien sus recursos" (Camacho 2012, 13), situación que fue muy cuestionada por diversos actores políticos de la Universidad y el estudiantado de la época, que fue el principal grupo en levantar la bandera por la autonomía. Ante tal situación, Daniel Camacho $(2012,14)$ expone que:

Todas las expresiones ideológicas, políticas, filosóficas y religiosas, incluidas aquellas muy afines al Gobierno, cerraron filas del lado universitario. Quienes conozcan a los universitarios de la época, podrán identificar a personas no solo afines, sino dirigentes importantes del partido go-
11. Esta "lucha desgarradora", en el contexto de la negociación del FEES para el año, según menciona González (2011, 35), el "Consejo Nacional de Rectores ha enfrentado en conjunto ciertas intromisiones o intentos directos de restringir los fondos de funcionamiento, lo que se considera una clara lesión a la autonomía universitaria", que además muestra lo complejo de este aspecto de la política universitaria. 
12. En 1970 , ocurrió la lucha contra la compañía Aluminum Company of America (ALCOA), debido a su intento de explotación de bauxita y cuyo contrato-ley en la Asamblea Legislativa movilizó a miles de estudiantes universitarios y de secundaria, quienes, en varias protestas, en especial la del viernes 24 de abril del mismo año, presionaron para que el contrato fuera desestimado. Para detalles ver: Alvarado, Raúl. 1998. "Jornadas de ALCOA", Revista Herencia, 13(1): 117-127. bernante, proponiendo y defendiendo, dentro del III Congreso, tesis en pro de la autonomía. Desde unos años antes, el clima en la Universidad de Costa Rica era de intensa agitación intelectual. Todo ello muestra, como se dijo antes, que el III Congreso es producto de un vigoroso movimiento de defensa de la autonomía universitaria.

Dicho clima en defensa de la autonomía universitaria se estableció de la mano con la organización social y política emergente en esa década de $1970^{12}$, que terminaría con reformas en el grado de la organización política universitaria y la reforma total del Estatuto Orgánico de la UCR en marzo de 1974. En este se establecieron la Asamblea Universitaria, el Consejo Universitario, la Rectoría, las Vicerrectorías, y la apertura de cargos políticos en las sedes, facultades y escuelas (Camacho 2012, 15).

Este artículo de Daniel Camacho conecta la acción universitaria, docente y estudiantil con las características históricas que la autonomía universitaria ha ido adquiriendo mediante cambios sustantivos en relación con los hechos que le suman o que le restan.

En cuanto al mismo tema, en la historia reciente de Costa Rica ha existido una serie de episodios en los que la autonomía universitaria ha sido puesta en cuestión y han existido pronunciamientos de la Sala Constitucional (Sala IV). Por ejemplo, se encuentra el trabajo del sociólogo y abogado Jorge Enrique Romero Pérez, titulado El derecho constitucional y la Universidad de Costa Rica (2008). Romero-Pérez $(2008,118)$ repasa las resoluciones de la Sala Constitucional (votos 4823-93, 6681-93, 200-94, 1306-94 y 3655-97) y las recapitula de la siguiente manera:

1. La autonomía de las universidades públicas es completa, especial, diferente y privilegiada, respecto de las demás instituciones públicas descentralizadas.

2. Los ámbitos de esa autonomía atañen a los aspectos administrativos, políticos, organizativos y financieros.

3. El subsistema institucional y jurídico de las universidades públicas está dentro del Estado y del ordenamiento jurídico nacional. No se trata de "un Estado dentro de otro Estado", sino de una situación en la cual (por ejemplo) el Poder Judicial puede intervenir cuando las autoridades universitarias violan la Constitución Política.

Sin embargo, Romero-Pérez (2008) no es el único que retoma los alcances en la discusión de la autonomía universitaria cuando ha sido necesaria la interpretación de la Sala Constitucional en momentos en que las universidades han sido presionadas por otros poderes del Estado para actuar u omitir alguna acción. También se encuentra la mencionada tesis de Vladimir Villalobos González (2005), en la que se estudian ocho casos en los que la Sala Consti- 
tucional ha fallado favorable a la autonomía universitaria, generando jurisprudencia relacionada.

En las resoluciones constituciones surgidas entre 1990 y el 2004, estudiadas por Villalobos (2005), se explica la relevancia de tales ratificaciones relativas a la "potestad legislativa frente la autonomía universitaria" y la "potestad jurisdiccional frente la autonomía universitaria", que entran directamente en el tema del equilibrio de poderes de la República y el garante e independencia de estos en relación con las universidades estatales del país.

El análisis presentado por Vladimir Villalobos González evidencia los niveles de conflicto alrededor de la institucionalidad de la autonomía universitaria y, por ende, de todo un espectro complejo de relaciones jurídicas. Sobre este contenido de relaciones jurídicas, una significativa y amplia referencia dedicada a esta temática se encuentra en el libro Defensa de la Autonomía Universitaria de Jorge Enrique Romero-Pérez, del 2010.

La publicación surgió en el contexto del suceso del 12 de abril del 2010, cuando el Organismo de Investigación Judicial (OIJ) ingresó sin autorización al campus de la Sede Rodrigo Facio de la UCR, so pretexto de buscar a un individuo en aparente fuga. Dicho evento suscitó una respuesta inmediata por parte del departamento de seguridad, funcionarios, docentes y estudiantes de la sede, que al ser testigos de la entrada sin autorización de funcionarios del OIJ, defendieron la autonomía universitaria, impidieron el ingreso ${ }^{13}$. Esta violación de la autonomía universitaria produjo que la UCR declarara el 12 de abril como el Día de la Autonomía Universitaria.

Con este balance sobre las condiciones de la reforma universitaria desde el contexto particular de Costa Rica, se pueden sugerir líneas de análisis y visualizar parte de los escenarios de acción y tensión que las universidades públicas y la autonomía universitaria presentan desde su constitución de actores sociales interrelacionados con otras instancias de poder estatales.

A partir de tal discusión, se pasará a focalizar más sobre los pilares propios y fundamentales que constituyen la reforma universitaria como legado histórico y político precedente y vigente en la región.

\section{Ámbitos de la autonomía universitaria latinoamericana}

Como se ha explicado, y, en el contexto de los 100 años de la Reforma Universitaria de Córdoba, es necesario comprender la continuidad práctica del legado de autonomía como un proyecto que intrínsecamente contiene las proclamas de cogobierno estudiantil, libertad de catedra, financiamiento propio, libertad política, protección jurídica y lucha social.

De esta manera, su constitución como proyecto centenario está representada por temporalidades en las que se proclamaba su instauración, se defiende su posición frente a otros actores sociales y se consolida su propia existencia. Por eso, la reforma universitaria también es su pasado de creación como lucha social en el siglo XX y talante en la construcción de las sociedades actuales en el siglo XXI. Es una situación que el investigador Armando Zam-
13. Para conocer el detalle y la implicación de este suceso, visitar:

http://www.ts.ucr.ac.cr/ua/auton omia.php 
brano Leal (2008) ha sintetizado en su artículo El movimiento de Córdoba: la mirada, el tiempo y la distancia, en el que afirma:

Casi cien años después de la grandeza y de su invaluable acierto, Córdoba nos convoca una vez más. No lo hacemos para trazar una forma de historia formal, tampoco para hacer de este movimiento único en América Latina un objeto de estudio y de investigación tradicional. Volvemos a él para encontrar nuevamente los dolores que nos aquejan, las angustias que vivimos, el desamparo en el que nos encontramos sumidos millones y millones de latinoamericanos. Volvemos a él para comprender sus efectos, sus alcances, sus logros. Regresamos a él en el tiempo, en la distancia, buscando comprender lo que aún nos sigue siendo negado. (Zambrano 2008, 228).

La reforma universitaria es una aliada a las formas de organización y transformación de cada país del continente. Así, desde la lectura global de Zambrano $(2008,227)$, hubo y hay un "Espíritu de 1918" expandido por toda Latinoamérica. Por tal motivo, la percepción de una gran incidencia histórica de la autonomía universitaria es una tesis defendida y asumida por los interesados en la materia y por los mismos actores políticos universitarios que han devenido en un continuum sucesorio de autonomías, en plural, según lo referencia la -ya citada- catedrática mexicana Renate Marsiske (2004, 162), quien refuerza el argumento de que entre:

1918 y 1929 como los de la reforma universitaria en América Latina, considerando que las formas de lucha estudiantiles fueron parecidas en todos los países, pero sus resultados diferentes. El movimiento de reforma iniciado en la Universidad Mayor de San Carlos y Montserrat de Córdoba, Argentina, en 1918 proyectó su influencia a los centros universitarios de toda América.

La referencia a este periodo engloba el proceso como un fenómeno que se fue replicando en cada país de Latinoamérica, en diferentes dimensiones y según la propia motivación de lucha de las generaciones juveniles como actores pioneros de los derechos políticos universitarios y la consecuente pro- 
clamación de la universidad pública como un espacio autónomo desde el mismo centro de sus aulas.

De ese modo, los procesos de lucha ligados a la autonomía universitaria han sido orientados hacia distintos ámbitos del espacio universitario, la relación entre universidad pública y sociedad, y los derechos de participación política de las juventudes universitarias. Esta condición en el acceso de derechos es la idea central del artículo Autonomía y derechos universitarios, del investigador mexicano Leonicio Lara Sáenz $(2010,124)$ y que expone las siguientes cualidades de la autonomía universitaria:

la libertad de cátedra de pensamiento y de investigación; la capacidad de gobernarse; la elección interna de autoridades; la personalidad jurídica propia de la institución, así como su propio patrimonio; la capacidad de establecer normas jurídicas internas obedientes del orden jurídico nacional y la capacidad de organizar sus propios planes y programas de estudio y expedir títulos profesionales, así como la libertad y autonomía de gestión y administración.

En este listado de ámbitos constituyentes de la autonomía universitaria se evoca la misma concepción de universalidad de estas instituciones de educación superior y ser espacios de intercambio sociocultural de los estudiantes que provienen de diferentes zonas de cada país, tanto urbanas como rurales.

Lo mismo sucede con el personal docente y cada sector administrativo de la universidad pública, que permite, además, el ascenso laboral y profesional de sus trabajadores. Por lo tanto, la autonomía universitaria es también una estructura laboral que mantiene alternabilidad política en sus dirigencias oficiales, en que cada sector, docente, administrativo y estudiantil, puede identificarse en un espacio asambleario que permite el intercambio entre clases, generaciones y marcos socioculturales (Lara 2010, 125).

Con lo expuesto anteriormente se comprende que la institucionalización de la autonomía universitaria está presente en los derechos en cada sector interno, llevando consigo una complejidad de ámbitos que constituyen las autonomías universitarias y que Lara Sáenz (2010, 125-126) explica diferenciadamente:

$\checkmark \quad$ La autonomía académica y técnica: referida a la competencia y capacidad para educar, organizarse académicamente, establecer programas y modalidades de estudio, así como facultad de expandir títulos profesionales.

$\checkmark$ La autonomía de gobierno: que comprende "la facultad y responsabilidad de gobernarse; la facultad de nombrar a sus autoridades internas; y la capacidad de sus miembros para participar en los ór- 
ganos de gobierno... relativo a la libertad para gobernarse, la libertad para nombrar internamente a sus autoridades y la participación de la comunidad en los órganos de gobierno y los procesos electorales participativos de la comunidad para realizar las designaciones".

$\checkmark \quad$ La autonomía legal, administrativa: desde el que se puede "detentar personalidad jurídica en cuanto persona moral de organismo descentralizado del Estado. Establecer su normatividad y reglamentación interna. Contar con patrimonio propio y administrar libremente el mismo".

$\checkmark \quad$ La autonomía financiera: Que se basa en la posibilidad de "recibir aportaciones financieras del Estado y destinar, disponer y comprobar pública y externamente el uso de tales aportes económicos, así como generar ingresos propios, sin alterar sus fines, y usar y disponer de los mismos en razón del cumplimiento de sus programas (...) al patrimonio de las universidades (...) créditos y presupuesto, y todo derecho y obligación cuantificable en recursos propios de la universidad".

Esta lectura desagregada planteada Lara Sáenz, comprende las condiciones para que las organizaciones estudiantiles sean legítimas y legales representantes políticas integrantes de la autonomía universitaria en cogobernanza con las autoridades políticas universitarias, hecho posible desde el marco de los derechos universitarios.

\section{Los derechos universitarios}

Tal concepción de derechos universitarios implica diferenciar el sector estudiantil en sus dos componentes: el estudiantado individual y estudiantado agrupado. El primero se relaciona con los derechos "a ser considerado alumno de la Universidad; a utilizar las bibliotecas; a igualdad de género; a ser respetado; a realizar actividades deportivas y utilizar las instalaciones de las mismas [sic]", explica Lara (2010, 129).

Mientras tanto, el estudiantado agrupado se sitúa en los diferentes espacios (asociaciones, sociedades y federaciones) del movimiento estudiantil; se relaciona con las acciones políticas de las agrupaciones estudiantiles reproduciendo los derechos "a votar; a ser votado; derecho de petición; derecho a presentar quejas o reclamaciones o denuncias", entre otras varias (Lara 2010, 129).

Venido de lo anterior, y por ser la autonomía universitaria una expresión político-jurídica de amplio alcance social desde el marco normativo, institucional y jurídico del Estado, el trabajo de Raúl Domínguez Martínez (1997), Autonomía universitaria: el jus abutendi de un concepto, hace un abordaje históricojurídico de la autonomía universitaria, tomando el caso la realidad mexicana. 
En su análisis, advierte que los grados de autonomía en las universidades mexicanas presentan una diferencia entre la que existe para el caso de la Universidad Nacional Autónoma de México (UNAM) y el de las universidades en cada Estado mexicano, debido al carácter nacional de la primera (Domínguez 1997, 50).

Empero, en todas estas, el carácter de autonomía les es dado desde la misma Constitución Política de México (así como en varias otras del continente). La situación explica el estamento político-jurídico dado por el Estado, sobre lo que el autor explica diciendo que "la autonomía que suele conceder el Estado consiste en la apertura de ciertos márgenes de libertad, pero no en ruptura" (Domínguez 1997, 51).

La autonomía cruzada de relaciones institucionales político-jurídicas estatales consolida sus propios grados de autonomía política, docente, estudiantil y administrativa comentados anteriormente por Lara Sáenz (2010), que, desde la lectura de Domínguez Martínez (1997), coexisten en el marco de una autonomía basada "en la capacidad jurídica" universitaria como institución abocada a la educación. No obstante, las capacidades de gestión y dirección política autónoma de las universidades tienen, según Domínguez (1997, 57), posibilidades de disminuir en cuanto al aspecto financiero:

La autonomía, cuando no incluye la autonomía financiera, resulta no ficticia, pero sí marcadamente restringida; y cuando sí la incluye, depende de cuál sea su grado de participación en el proyecto dominante o el grado de absorción que el proyecto dominante tenga respecto de su potencial disidente.

Con base en ese criterio, el autor cuestiona la práctica real de la autonomía en una expresión mayor que se pueda mantener estable en el tiempo ${ }^{14}$. En este escenario, Luis Fuenmayor (2008), partiendo de la experiencia venezolana en Autonomía universitaria y reforma constitucional, explica que la primera se define desde el ejercicio mismo de esta condición:

La autonomía sola no describe a la universidad. Existen muchas instituciones autónomas que no son universidades. La autonomía es una cualidad que se otorga o se adquiere, por lo que no tiene existencia por sí misma, sino a través [sic] de la institución que la posee, la ejerce y la disfruta (Fuenmayor 2008, 119).
14. Por este motivo, Domínguez (53-68) amplía la discusión desde las etapas de la historia de la Universidad Nacional Autónoma de México (UNAM). Se refiere al contexto inicial de esa Universidad en la década de 1920; los años cercanos a los movimientos estudiantiles de 1968; la etapa de reconversión en el contexto de neoliberalización económica; y el contexto actual en que modelos empresariales ejercen presión en las universidades públicas y, por ende, en su autonomía. http://www.ts.ucr.ac.cr/ua/auton omia.php

Según Fuenmayor (2008), la práctica institucional de la autonomía define su alcance y las cualidades con respecto a su actuación con otras instituciones 
15. Diversos han sido los casos en los que las fuerzas estatales de Gobierno han violentado la autonomía universitaria ocupando el espacio físico del campus como muestra de dominio. Entre otros casos, Guatemala, 1944; México, 1968; El Salvador, Argentina y Chile, 1973; Honduras, 2009; y Nicaragua, 2018. sociales o políticas que componen los Estados modernos; de ahí su carácter histórico en sí mismo, más allá de las propias concepciones y gestiones en el seno de las universidades.

Estos niveles de gestión y política autónoma son formas en las que esta existe y se garantiza a expensas de la presión directa de otros sectores estatales, sociales o políticos. Precisamente, una de las manifestaciones de más fortaleza de autonomía universitaria se encuentra en la inviolabilidad de sus recintos o campus universitarios, sobre lo que Ramón Calzadilla $(2002,2)$ en Ética y autonomía universitaria, explica que:

La inviolabilidad de los recintos universitarios es parte integrante de la autonomía universitaria; sin embargo, debe ser concretada de manera separada. Consiste en el derecho de la universidad de conservar y hacer respetar la intangibilidad de sus superficies de terreno y locales en donde despliega sus actividades. No se trata de extraterritorialidad.

Esta afirmación de que la autonomía del campus universitario "no se trata de extraterritorialidad" radica en la diferencia entre autonomía e independencia: el campus es un espacio de control administrativo y restringido del que gozan las universidades públicas en América Latina, con la posibilidad de resguardar el orden, los sistemas de seguridad, los recursos, los activos y a las personas que las integran.

La autonomía del campus universitario se coloca como uno de sus elementos más distintivos. Es una de las expresiones más palpables y defendibles, así como el espacio que diversos Gobiernos autoritarios del continente han traído abajo al introducir al Ejército, a la Policía Nacional o a las fuerzas secretas de seguridad $^{15}$.

Por otra parte, y como preámbulo a la siguiente sección sobre los retos de la autonomía universitaria, Calzadilla (2002) discute sobre las formas en que se presiona la autonomía desde lógicas de poder estatal con miras a fines políticos que se expresan desde las ya mencionadas formas de intervención directa de las fuerzas armadas, así como amenazas de reducción presupuestaria lo que en ocasiones ha llevado a que las universidades realicen cambios curriculares, políticos, administrativos o de matrícula.

\section{En su centenario: retos de la autonomía universitaria hoy}

Este contexto centenario de la reforma, el cogobierno estudiantil y la autonomía universitaria entre 1918 y el 2018, implica, a su vez, una proyección hacia el futuro según los desafíos actuales y los que estarían por venir. En ese 
sentido, varios autores han planteado lo que denominan los actuales retos de la autonomía universitaria.

Una de estas reflexiones surgida desde Perú por el investigador Esteban Eduardo Zárate Cárdenas, en su artículo Autonomía universitaria ayer y hoy (2008), apunta al reto de mantener la autonomía universitaria de las universidades públicas en un "contexto de privatización de la educación superior".

Además, señala que es necesaria otra nueva reforma que profundice los alcances de la antecesora centenaria y se separe del contexto económico actual "donde se respete el derecho a la comunicación, la democracia, las libertades, el derecho a la diferencia, la inclusión, la equidad y la no discriminación" (Zárate 2008, 3).

El autor señala que las políticas de privatización de varios sectores, incluido el de educación superior, ha generado condiciones de inestabilidad para la propagación legitima de la autonomía universitaria, ya no solo del Estado y la Iglesia, sino del mercado también. Ante tal situación, Zárate $(2008,4)$ reflexiona que están cambiando los alcances de las universidades públicas para cumplir su tarea de elevar la cultura, propagar investigaciones y ampliar el conocimiento científico en las sociedades.

Del mismo modo, Zárate expresa que los retos de las universidades públicas son continuar la investigación, la difusión de la cultura, la proyección social y una docencia generadora de conocimiento, desde escenarios autónomos y propios de la conciencia crítica que la universidad ha tenido.

En esta línea de análisis, pero desde México, el exrector de la UNAM, José Narro Robles, en su artículo La Autonomía Universitaria en el Bicentenario y sus perspectivas (2011), explica que los desafíos desde las universidades públicas se deben trazar desde los contextos de desigualdad que afectan toda la región de América Latina.

Narro $(2011,15)$ plantea que "la tarea fundamental de cultivar el saber define entonces a la universidad"; y también "la autonomía universitaria implica la fidelidad a la misión académica", lo que lleva consigo un posicionamiento sobre las razones de ser la autonomía universitaria y, por ende, de los retos actuales por preservar. En cuanto a este tema, el autor invita a la defensa de la autonomía universitaria como defensa de la libertad humana desde una amplia reflexión:

La tarea fundamental de cultivar el saber define entonces a la universidad como organización y espacio social que tiene como eje de sustentación la cultura, requiere de libertad como condición necesaria para realizarla; libertad de pensamiento, de búsqueda de la verdad y de expresión (...). El hecho de que una institución sea autónoma, en alguna forma, al menos deseable, implica que las per- 
sonas que la constituyen también lo sean o que lleguen a serlo (Narro 2011, 16).

Las anteriores consideraciones del exrector de la UNAM superan las nociones técnicas de la autonomía universitaria y las lleva a un nivel filosófico y fundamental sobre esta, al señalarlas como una construcción permanente de sí misma, y que ha existido y persiste debido a la ejecución que sus actores internos realizan, actúan y luchan.

Narro Roble $(2011,17)$ señala que "la autonomía no se adquiere de una vez y para siempre; se consigue con su ejercicio cotidiano en un proceso siempre inacabado". Sobre tal situación concluye que los retos actuales de la autonomía universitaria se centran en tres elementos medulares: "el compromiso social de las universidades, su financiamiento y la cobertura de la educación superior" (Narro 2011, 18-20).

En sincronía con lo recién analizado, el doctor en derecho de la Pontificia Universidad Católica del Perú, Marcial Antonio Rubio Correa, en su artículo La autonomía universitaria en el siglo XXI (2011), enfatiza en el pilar de construcción de conocimiento como eje central sobre el que descansan la reforma y la autonomía universitaria. Él deja en claro que este solo surge y se reproduce desde contextos en los que haya condiciones democráticas, sin caer en lógicas empresariales que busquen fines productivos, lo que a la vez implica una conciencia sobre la "la incorporación social a la universidad" (Rubio 2011, 41).

El trabajo de Rubio Correa surge de su vinculación con la Unión de Universidades de América Latina y el Caribe (UDUAL) como plataforma político-reflexiva que trabaja sobre la educación superior. Entonces, las reflexiones de este autor apelan directamente a lo que este organismo internacional estableció en su cumbre y declaración del 26 de noviembre del 2010. Como retos actuales, se establecieron los siguientes objetivos de la autonomía universitaria:

1. Gobierno de la universidad por sí misma

2. Realización de sus fines de educación, investigación y cultura

3. Respeto de su libertad de cátedra

4. Respeto de su libertad de investigación

5. Respeto del libre examen

6. Respeto de la libre discusión de las ideas

7. Libertad universitaria para determinar sus planes y programas

8. Libertad para fijar términos de ingreso, promoción y permanencia del personal

9. Libertad de administrarse a sí misma, en términos económicos amplios 
Este conjunto de objetivos perfilados como retos por la UDUAL y reproducidos por Rubio, apunta a la ratificación de las proclamas del "Grito de Córdoba" de 1918. Este es el inicio y pilar fundamental por sostener y reforzar en el contexto de su centenario. En esa línea, las condiciones de los modelos de competitividad devenidos desde las lógicas de neoliberalización económica, que también llegan a las universidades públicas y la educación superior en general, hacen que la acreditación no sea posible de esquivar. No obstante, Rubio $(2011,42)$ defiende que se debe y se puede llevar a cabo para el beneficio mismo de la autonomía universitaria. En sus palabras:

La acreditación universitaria es un proceso que bien llevado engrandece a la universidad. Pero si la acreditación se tuerce por presiones políticas o intereses burocráticos puede ser un instrumento de sojuzgamiento de las universidades. No estamos en la alternativa de aceptar o no aceptar los procesos de acreditación. Debemos entrar en ellos; pero, al mismo tiempo, debemos ser activos en perfilarlos y discutirlos creativamente. Durante el siglo XXI, una acreditación mal llevada puede conducir a la eliminación de la autonomía universitaria y afectar todos los aspectos de la vida institucional.

En última instancia, y como punto en común entre diversos autores anteriormente analizados, se encuentra la urgencia por luchar para que las universidades "produzcan relaciones financieras seguras, equitativas y previsibles entre el erario y la economía universitaria. Empero, debemos también asegurar nuestros propios ingresos, que son una de las bases más importantes de la autonomía. Ante tal situación, el autor sentencia que "nuestro financiamiento integral como instituciones universitarias debe ser repensado" (Rubio 2011, 43).

En ese sentido, la cercanía analítica del conjunto de autores recién citados, lleva a comprender la actualidad de la autonomía universitaria como una urgencia por atender los asuntos financieros y la defensa del propio campus que sigue siendo un espacio determinante en esta discusión. Por consiguiente, el artículo El reto de la autonomía universitaria (2011) de la exrectora de laUCR, Yamileth González García, agrega que:

Si queremos fortalecer la participación y ampliar el acceso; si queremos conservar la capacidad crítica; si queremos tomar nuestras decisiones sin presiones políticas ni económicas, las universidades debemos defender nues- 
tra autonomía, nuestra posibilidad de decidir y de proponer cambios (31).

Lo señalado por González García es sincrónico con lo anteriormente planteado por Narro Robles sobre la "responsabilidad" de hacer efectiva la lucha de protección y continuidad de la Reforma. De ahí que la retrospectiva sobre las concepciones de la autonomía universitaria lleva a las prospectivas de esta, ante lo cual la autora González $(2011,31)$ se posiciona al afirmar que:

Mantener la autonomía es darse la posibilidad de actuar con libertad y manejar el gobierno universitario sin ninguna intromisión (...) Defender la autonomía es poder construir creativamente, crear sin censura y transformar en conjunto lo que se considere necesario (...) Es decir, la autonomía no significa negar una fiscalización adecuada posterior a las acciones, sino rechazar la desaprobación o la censura establecida de antemano.

De esta manera, la autonomía tanto en perspectiva como prospectiva, puede ser matizada desde las posiciones que sobre esta se defiendan y manifiestan como parte del complejo aspecto de visualizarse como "la" filosofía de las universidades públicas y uno de los pilares que le dotan de sentido, significado y motivación.

\section{Conclusión: defender la autonomía sin caer en el aislacionismo}

Las condiciones actuales de la educación superior universitaria dadas en contextos globales de políticas de privatización macroeconómica, han generado un aumento en las expresiones privadas de la oferta de la educación superior; algunas incluso, con matices de lucro desde la transnacionalización empresarial desde dichas instituciones de estudios superiores. Dicho contexto ha llevado a una respuesta ampliada por quienes han construido una vida profesional desde la reflexión sobre la educación superior y autonomía universitaria.

Uno de estos exponentes es el investigador nicaragüense Carlos Tünnermann Bernheim, que en varios momentos ha buscado reforzar el conocimiento y la política relacionada con la educación superior pública y autónoma. Entre sus publicaciones, se encuentra La autonomía universitaria en el contexto actual, del 2006.

Aparte de plantear un recuento histórico de los orígenes de las universidades desde los modelos de Bolonia, París y Salamanca creados en el medioevo, 
recoge las peculiaridades de la universidad autónoma latinoamericana que en 1918 rompió con la universidad colonial hispánica, señorial y clasista que servía a los intereses de la Corona, la Iglesia y las clases superiores de la sociedad (Tünnermann 2006, 23).

Esta vinculación entre autonomía universitaria y política universitaria es una consecuencia propia de las bases de la educación superior pública latinoamericana, que permite construir espacios de discusión continentales.

Entre estos, el Foro Internacional sobre Autonomía Universitaria, auspiciado por la Asociación Colombiana de universidades (ASCUN), en junio del 2004, según señala Tünnermann $(2006,28)$, quien concluyó que:

La autonomía universitaria es uno de los temas más sensibles para la universidad, en el marco de las relaciones que ella mantiene con los demás actores sociales. En el mundo académico, considerado por muchos como el "tercer sector", este cuidado por preservar, e incluso defender, el carácter autónomo de la universidad ha sido tarea constante a lo largo de su historia y para cada momento, ante diversos actores que han aplicado fórmulas de intervención también distintas, al igual que los argumentos esgrimidos; pero con la constante de limitar las posibilidades de la acción universitaria, buscando subordinarla a fines que le son ajenos.

16. Tünnermann explica sobre la oposición a la OMC que, entre otros temas, han hecho manifiesta "la Asociación Internacional de Universidades (AIU), la Unión de Universidades de América Latina y el Caribe (UDUAL), la Asociación de Universidades y Colegios de Canadá, la Asociación de Universidades Europeas, el American Council on Education, la Asociación de Universidades del Grupo de Montevideo, el Consejo Superior Universitario Centroamericano (CSUCA) y las Cumbres Iberoamericanas de Rectores de Universidades Públicas" (Tünnermann 2006, 38). 
Tampoco es aceptable la pretensión de declarar a la educación superior como "un bien público global", pues el calificativo de global se presta a que no esté sujeta, en cada Estado, a las normas y regulaciones que soberanamente establezca cada país; y es una manera sutil de empujar a la educación superior a la órbita de la OMC, sacándola de la jurisdicción de los Estados nacionales, y, por lo mismo, renunciando estos a toda regulación o normativa que impida su libre circulación y comercio.

Así pues, las referencias de Carlos Tünnermann, en relación con los retos actuales y contemporáneos de las universidades públicas, cierran con una reflexión que engloba la necesidad de seguir defendiendo la autonomía universitaria como el camino para repensarla como baluarte continental y de esperanza para las sociedades contemporáneas:

La autonomía no implica aislamiento, sino presencia en el ámbito nacional e internacional, fortalecimiento de nuestros valores culturales y de nuestra identidad como naciones; pero abiertas al diálogo intercultural y a la comunidad académica internacional. Solo siendo instituciones libres y responsables, las universidades pueden ser las interlocutoras por excelencia de ese respetuoso y fructífero diálogo internacional. En este mundo contemporáneo, pletórico de desafíos e incertidumbres, la Universidad necesita autonomía, incluso para replantearse su propio ser y quehacer. (Tünnermann 2006, 44).

Como se ha venido explicando, las autonomías universitarias latinoamericanas han pervivido durante un siglo, pese a diversos lapsos de cuestionamiento por poderes políticos y han sabido reestructurarse sin perder las proclamas iniciales de la lucha universitaria de Córdoba de 1918.

Sobre ello, el investigador Axel Didriksson, en La autonomía universitaria desde su contemporaneidad (2006), argumenta que en la actualidad la autonomía universitaria se enfrenta a "nuevas hegemonías", tanto en el carácter epistémico como, centralmente, en el campo político (Didriksson 2006, 4-10). 
Tal carácter de continuidad de la autonomía universitaria en el contexto global de las fuerzas estatales latinoamericanas lleva a considerar con primacía la necesidad de seguir proclamando la autonomía universitaria como vector democrático en la pluralidad de actores sociales presentes en el continente. Entonces, citando a Didriksson $(2006,13)$, es necesario refrescar que:

La Universidad no ha sido ni será un reflejo de la lucha por el poder o por la reconformación de las relaciones con el Estado, como si fuera una institución que refleja solo fuerza externas a su entorno; más bien se trata de una institución de tal fuerza y magnitud que no es una simple variable dependiente de la construcción permanente de fuerzas de un determinado Estado, sino una entidad social que determina y a veces sobredetermina la conciencia social, la identidad cultural e histórica de un país y la solución de un determinado conflicto o el antagonismo social profundo.

Finalmente, y siguiendo con estos planteamientos del trabajo colectivo de José Narro, Martiniano Arredondo, David Moctezuma, Juan Aróstegui y Luis Raúl González, llamado Perspectivas y retos actuales de la autonomía universitaria (2009), se refuerza la tesis de mantener estrecho el vínculo entre labor universitaria y necesidades de la sociedad. Para ello, en un acto de adhesión a los acuerdos de la Conferencia Mundial de Educación Superior de 1998, exponen que:

la educación superior debe reforzar sus funciones de servicio a la sociedad y más concretamente sus actividades orientadas a la erradicación de la pobreza, de la intolerancia, de la violencia, del analfabetismo, del hambre, contra el deterioro del medio ambiente y en oposición a las enfermedades, principalmente a través [sic] de un enfoque inter y transdisciplinario para analizar los problemas y las cuestiones planteadas (Narro et al. 2009, 77).

En esta lógica de contraposición de la autonomía universitaria frente las tendencias mercantilistas de la globalización, las universidades públicas están asediadas por intereses de sectores políticos que buscan su privatización y promueven la reducción de sus presupuestos por parte de los Estados. Ante 
ello, y en correspondencia con los autores citados (Narro et al. 2009, 79), es fundamental concluir que "la educación no puede en modo alguno, quedar regida por reglamentos e instituciones previstas para el comercio, ni por la lógica del mercado".

\section{Bibliografía}

Alvarado, Raúl. 1998. "Jornadas de ALCOA", Revista Herencia, 13 (1): 117127.

Baudrit Carrillo, Luis. (2005). Repensar la autonomía de la Universidad de Costa Rica. San José de Costa Rica: SIEDIN/UCR.

Baudrit Carrillo, Luis. (2009). Autonomía universitaria y control de la hacienda pública. San José de Costa Rica: SIEDIN/UCR.

Camacho Monge, Daniel. 2012. "La autonomía universitaria, la vigencia del III Congreso Universitario y una obligada referencia a Rodrigo Facio". Revista de Ciencias Sociales 0 (138). https://doi.org/10.15517/rcs.v0i138.12082.

Calzadilla, Ramón. "Ética y autonomía universitaria". Sapiens. Revista Universitaria de Investigación 3, núm. 2 (2002).

Cazali Ávila, Augusto. 1977. "La autonomía universitaria en Centroamérica". Anuario de Estudios Centroamericanos 3 (1): 9-26.

Didriksson, Axel. "La autonomía universitaria desde su contemporaneidad". Universidades, núm. 31 (2006): 3-16.

Domínguez Martínez, J. Raúl. "Autonomía universitaria. El jus abutendi de un concepto". Política y cultura, núm. 9 (1997). http://www.redalyc.org/html/ 267/26700904/.

Fuenmayor Toro, Luis. "Autonomía universitaria y reforma constitucional". Educere 12, núm. 40 (2008): 118-126.

Galdámez, Luis (1935). La universidad autónoma. San José, Costa Rica: Editorial Borrasé.

García de Paredes, Gustavo. 2008. "En defensa de la autonomía universitaria". Contenido Contenido, 10. 
González García, Yamileth. 2011. "El reto de la autonomía universitaria". Universidades 61 (49): 31-40.

Lara Sáenz, Leoncio. "Autonomía y derechos universitarios". Perfiles Educativos 32 (2010): 123-132.

Marsiske Schulte, Renate. "Historia de la autonomía universitaria en América Latina". Perfiles educativos 26, núm. 105-106 (2004): 160-167.

Naishtat, Francisco, Ana María García Raggio, Federico Schuster, y Susana Villavicencio. "Filosofía política de la autonomía universitaria". Perfiles Educativos, 1996. http://www.redalyc.org/articulo.oa?id=13207301.

Narro Robles, José, Martiniano Arredondo Galván, David Moctezuma Navarro, Juan Aróstegui Arzeno, y Luis Raúl González Pérez. "Perspectivas y retos actuales de la autonomía universitaria". Revista de la educación superior 38, núm. 152 (2009): 65-94.

Narro Robles, José. "La Autonomía Universitaria en el Bicentenario y sus perspectivas". Universidades 61, núm. 48 (2011): 14-20.

Romero Pérez, Jorge Enrique. 2008. "El Derecho Constitucional y la Universidad de Costa Rica: Reflexión en las circunstancias hasta 2009". Revista de Ciencias Jurídicas 0 (119). http://revistas.ucr.ac.cr/index.php/juridicas/article/view/9776.

Romero Pérez, Jorge Enrique. 2010. Defensa de la autonomía universitaria pública. 1. ed., Especial. San José, C.R: Univ. de Costa Rica.

Rubio Correa, Marcial. "La autonomía universitaria en el siglo XXI", 2011. http://iep.udea.edu.co:8180/jspui/handle/123456789/40.

Suárez Zozaya, María Herlinda. "Consideraciones políticas sobre la autonomía universitaria". Perfiles Educativos 32 (2010): 27-49.

Tünnermann Bernheim, Carlos. "La autonomía universitaria en el contexto actual". Universidades, núm. 31 (2006): 17-40.

Varela, Luis Muñoz, y Juan Félix Castro Soto. "El principio de autonomía universitaria entre Escila y Caribdis / Principle of university autonomy between Escila and Caribdis". Actualidades Investigativas en Educación 6, núm. 1 (el 28 de marzo de 2011). https://doi.org/10.15517/ aie.v6i1.9202. 
Villalobos González, Vladimir. 2005. "Autonomía universitaria y aplicación del artículo 84 de la Constitución Política de Costa Rica, caso Universidad de Costa Rica, período 1990 a 2004". http://repositorio.sibdi.ucr.ac.cr:8080/jspui/handle/123456789/1408.

Zambrano Leal, Armando. "El Movimiento De Córdoba: La Mirada, El Tiempo Y La Distancia". Educere, 2008. http://www.redalyc.org/articulo.oa? $\mathrm{id}=35611336001$.

Zárate Cárdenas, Esteban Eduardo. 2008. "Autonomía universitaria ayer y hoy". Universidades 58 (36): 91-94. 